\title{
Aberrantly Expressed Non-Coding RNAs in the Placenta and Their Role in the Pathophysiology of Gestational Diabetes Mellitus
}

\author{
Runyu Du (D) \\ $\mathrm{Na} \mathrm{Wu}$ \\ Ling Li iD \\ Department of Endocrinology, Shenging \\ Hospital of China Medical University, \\ Shenyang, Liaoning Province, People's \\ Republic of China
}

\begin{abstract}
Gestational diabetes mellitus (GDM), one of the most common complications during pregnancy, is associated with a high risk of short- and long-term adverse effects on the mother and offspring. Placenta-derived hormones and cytokines aggravate maternal insulin resistance (IR) during pregnancy, which in turn contribute to GDM. The hyperglycemia and IR in GDM result in aberrant placental structure and function adversely affecting fetal growth and well-being. Therefore, it is reasonable to assume that structural and functional alterations in the placenta contribute to the pathogenesis of GDM and GDMrelated complications. Increasing evidence suggests that multiple non-coding RNAs (ncRNAs), including microRNAs, long non-coding RNAs, and circular RNAs, are dysregulated in placentas of patients with GDM and linked to abnormal placental structure, metabolism, and function. Manipulation of ncRNA expression led to some key pathophysiological features of GDM, such as trophoblast dysfunction, changes in intracellular glucose metabolism, and inflammation. Moreover, placenta-specific ncRNAs may be potential diagnostic biomarkers and even therapeutic targets for GDM. This review summarizes data published on the involvement of aberrantly expressed placental ncRNAs in GDM and provides information on their role in the pathogenesis of GDM and GDMassociated complications.
\end{abstract}

Keywords: dysregulated ncRNA, pregnancy disorder, placental dysfunction, pregnancyrelated complications, trophoblast dysfunction

\section{Introduction}

Gestational diabetes mellitus (GDM), a metabolic disorder during pregnancy, is diagnosed in the second or third trimester and accounts for $86.4 \%$ of all hyperglycemia in pregnancy. ${ }^{1,2}$ Global increases in the incidence of obesity and maternal age have resulted in an increased incidence of GDM (approximately 14\% globally). Epidemiological studies have shown that the incidence of GDM in pregnancies worldwide varies from $9 \%$ to $21 \%$ owing to the diverse genetic backgrounds and epigenetic modifications that occur in response to nutritional and environmental factors. ${ }^{2}$ GDM can cause various complications in pregnant women and fetuses, including short- and long-term effects. ${ }^{3,4}$ However, the precise etiological mechanisms of GDM and GDM-related complications remain unclear, despite significant efforts over decades.

GDM is presumed to be a complication of pregnancy involving aberrant placental functions because the maternal metabolic status is rapidly relieved after the
Department of Endocrinology, Shengjing Hospital of China Medical University, No. 36, Sanhao Street, Heping District, Shenyang, Liaoning Province, II 0004 People's Republic of China

Tel +86 I894025 II8I

Fax +86 24-25944460

Email liling8864@hotmail.com 
expulsion of the placenta at delivery. ${ }^{5}$ It is known that insulin resistance (IR) is a hallmark of the pathogenesis of GDM. The development of IR in pregnancy is caused partly by placental hormones, such as estradiol, progesterone, prolactin, cortisol, human placental lactogen, and human placental growth hormone. ${ }^{6}$ Due to the very short half-lives of placental hormones, the glucose homeostasis is characteristically rapidly restored within 24 to 48 hours after delivery. ${ }^{7,8}$ For patients with type 1 diabetes, the insulin requirement is restored to pre-pregnancy levels, or even lower within 1 to 2 days after delivery. ${ }^{9}$ As an endocrine organ, the placenta also secretes adipokines and cytokines such as leptin, adiponectin, tumor necrosis factor alpha (TNF- $\alpha$ ), and interleukin-6 (IL-6), which accelerates the inflammatory response and in turn contributes to IR during pregnancy. ${ }^{6}$ Conversely, hyperglycemia and severe IR lead to alterations of the placental structure and function, thereby adversely affecting fetal growth and well-being. Based on aberrant villous vascularization, an imbalance of vasoactive molecules, and enhanced oxidative stress, the alterations in placental structure and function lead to impaired fetal oxygenation and changes in transplacental nutrient supply. ${ }^{5,10}$

RNAs without coding potential, referred to as noncoding RNAs (ncRNAs), account for the majority of RNAs. ${ }^{11}$ There are two major groups of ncRNAs: (1) linear RNAs, including microRNAs (miRNA) and long non-coding RNAs (lncRNA) (based on length), and (2) circular RNAs (circRNA) consisting of a "back-splicing" loop. ${ }^{11}$ miRNAs regulate target transcript expression through degradation or translational repression by incorporating into the RNA-induced silencing complex. ${ }^{12}$ Compared with our understanding of miRNAs, our understanding of the mechanistic characterization of lncRNAs and circRNAs is rather poor. lncRNAs can exhibit biological functions in the nucleus or cytoplasm. Nuclear IncRNAs primarily regulate chromatin, transcription, and variable splicing. Cytoplasmic lncRNAs act as sponges for other transcripts or proteins, serve as protein templates, or regulate mRNA degradation and translation. ${ }^{13}$ CircRNAs may regulate gene expression at multiple levels, such as sponging miRNA, influencing mRNA stability, or regulating transcription. ${ }^{14}$ Accumulated evidence indicates that ncRNAs play a role in pathway regulation and developmental and pathological processes. ${ }^{11,15}$ In a tissue-specific and temporal manner, the expression of multiple ncRNAs varies in human placenta during different stages of placental development in line with their functions in regulating placental development and trophoblast cell activities. ${ }^{16}$ Taking miRNAs for example, the C19MC and C14MC miRNA clusters are specifically or preferentially expressed in the placenta, and C19MC miRNAs expression in trophoblast cells increases substantially from the first to the third trimester, whereas C14MC miRNAs show the opposite trend. ${ }^{17,18} \mathrm{~A}$ number of ncRNAs exert regulatory effects on trophoblast cell proliferation, apoptosis, migration, and invasion as well as on metabolism in trophoblasts. ${ }^{19,20}$ These findings suggest that ncRNAs can regulate placental development and function. Microarray analyses and RNA profiling data indicate that multiple ncRNAs are dysregulated in placentas of patients with GDM. ${ }^{21-24}$ Several studies have explored the mechanisms by which ncRNAs participate in GDM. ${ }^{25,26}$ Conceivably, aberrant ncRNA expression can disrupt normal placental function, initiating the pathogenesis of GDM and GDM-associated complications.

This review summarizes recent evidence on the role of dysregulated ncRNAs in the placentas of patients with GDM. Understanding their functions might help elucidate the complex pathophysiological mechanisms in GDM and related complications and might be valuable in identifying novel therapeutic targets.

\section{Dysregulated Placental ncRNAs are Associated with GDM}

The aberrant structural and functional alterations of the placenta have been suggested as important contributory factors to the pathogenesis of GDM and GDM-associated complications. ${ }^{5,6}$ To date, numerous studies have demonstrated that placenta-specific miRNAs play critical roles in the pathogenesis of GDM, while few studies have investigated the role of placenta-specific lncRNAs and circRNAs in GDM. These studies will be discussed in the subsequent sections.

\section{Placenta-Specific miRNAs in GDM}

Several studies have evaluated the potential role of dysregulated miRNAs in the placentas of patients or animals with GDM (Table 1). To elucidate the role of specific miRNAs, it is necessary to identify the target mRNAs post-transcriptionally regulated by miRNAs. Several human studies showed that multiple miRNAs were differentially expressed in the placentas of GDM cases and controls; the expression levels of these miRNAs were negatively correlated with the protein and/or mRNA levels 


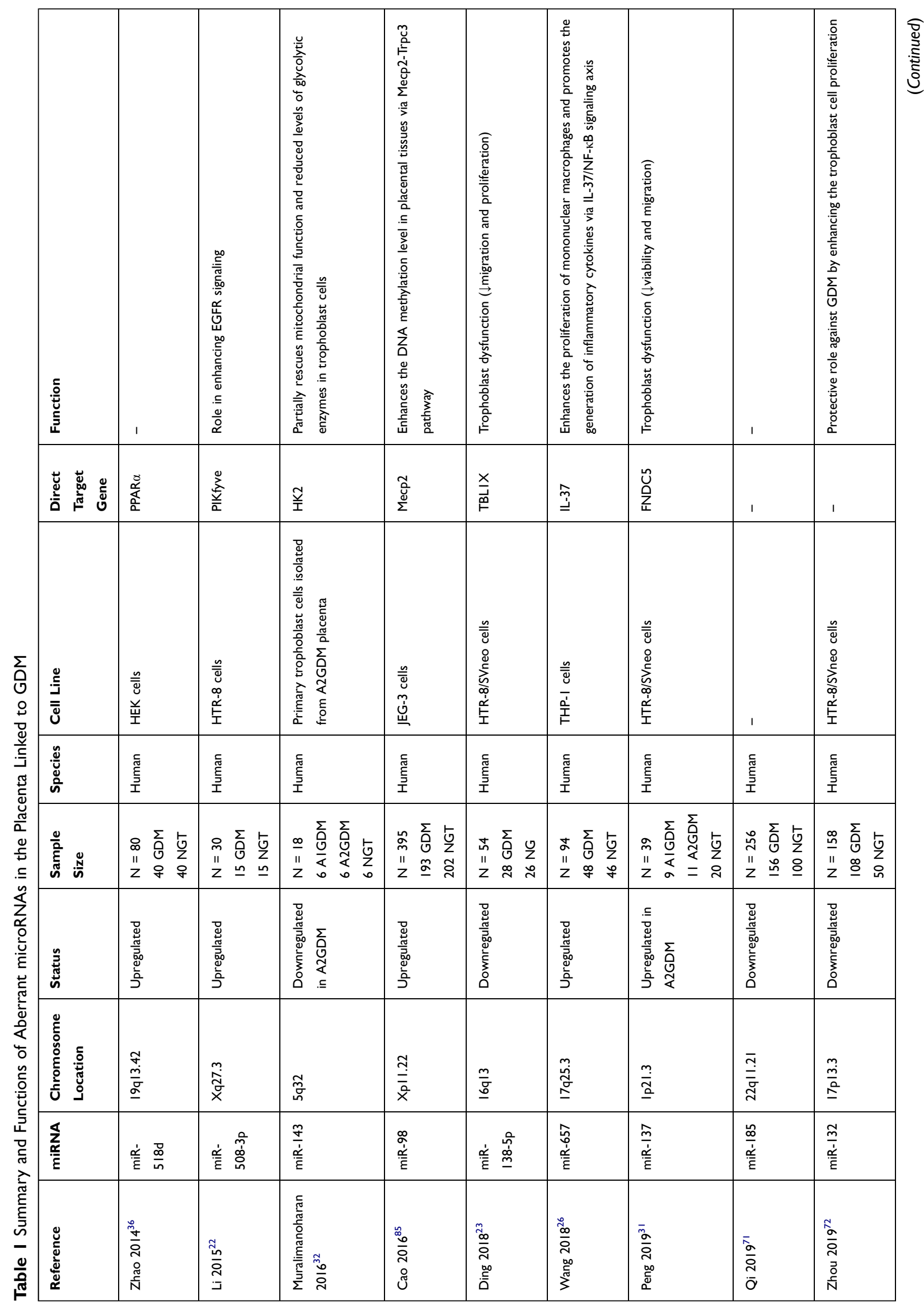




\begin{tabular}{|c|c|c|c|c|c|c|c|c|}
\hline 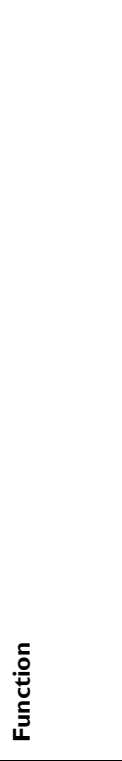 & 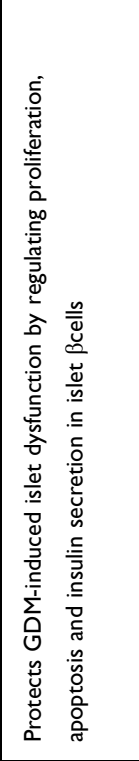 & 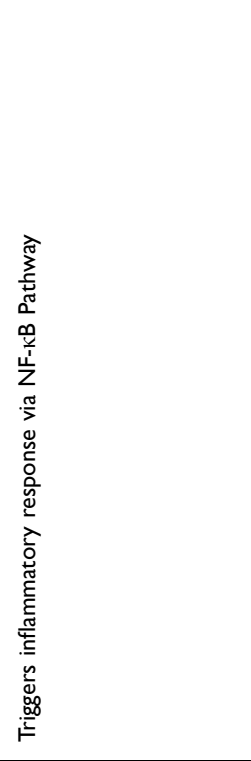 & 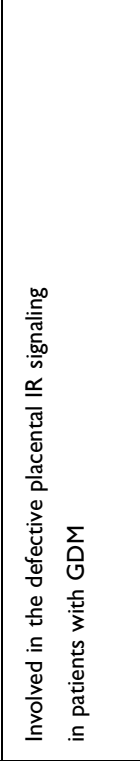 & 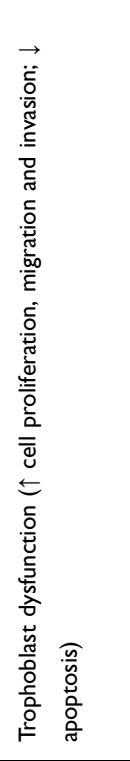 & 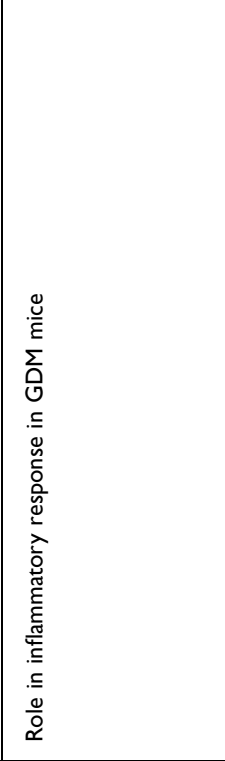 & 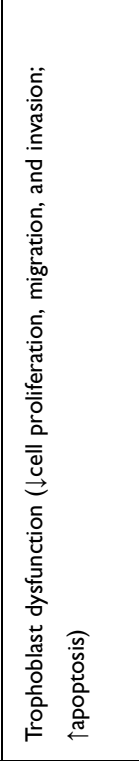 & 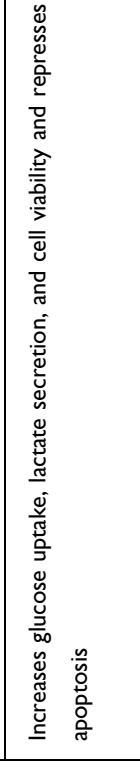 & 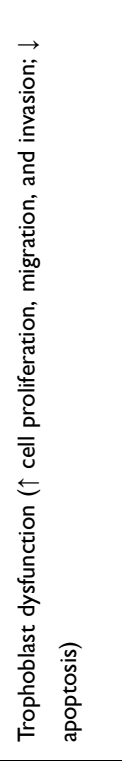 \\
\hline 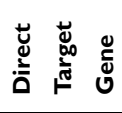 & $\frac{\bar{z}}{\alpha}$ & 颜 & $\begin{array}{l}\stackrel{s}{\dot{\leftrightarrow}} \\
\underline{\underline{u}} \\
\underline{\underline{u}}\end{array}$ & 离 & $\begin{array}{l}+ \\
\tilde{u} \\
0 \\
0\end{array}$ & 帝 & 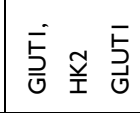 & 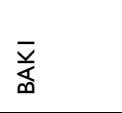 \\
\hline 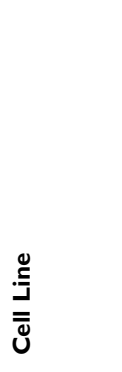 & $\begin{array}{l}\underline{\underline{\underline{E}}} \\
\frac{\dot{s}}{\underline{s}}\end{array}$ & 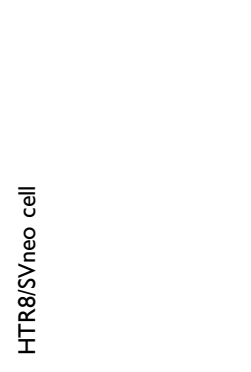 & 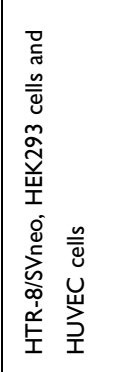 & 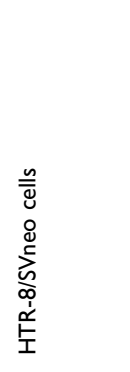 & 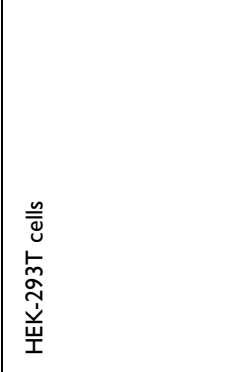 & 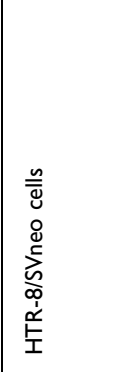 & 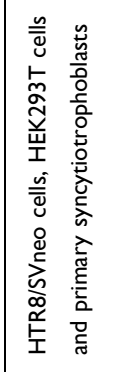 & 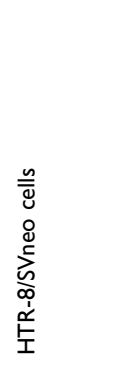 \\
\hline 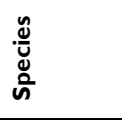 & \begin{tabular}{|l} 
\\
$\frac{y}{x}$ \\
\end{tabular} & 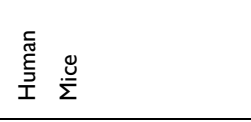 & 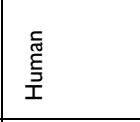 & 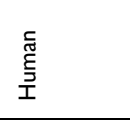 & 产 & 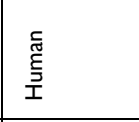 & \begin{tabular}{|l} 
离 \\
童 \\
\end{tabular} & 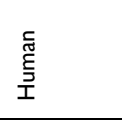 \\
\hline 递。 & 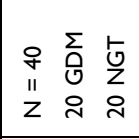 & 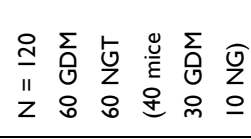 & 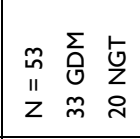 & 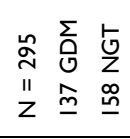 & 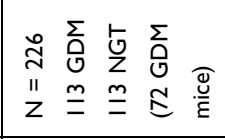 & 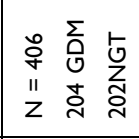 & 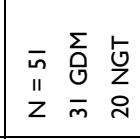 & \begin{tabular}{lll} 
& \multicolumn{5}{c}{} & 5 \\
0 & 0 & 0 \\
11 & 0 & 0 \\
$z$ & 0 & 0 \\
$n$
\end{tabular} \\
\hline 号 & 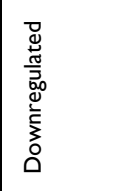 & 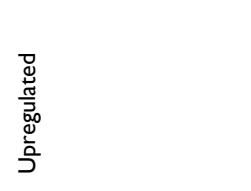 & 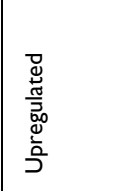 & 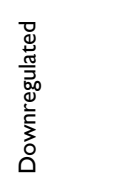 & 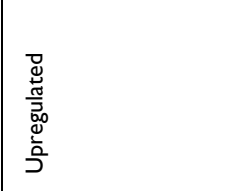 & 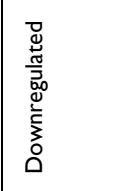 & 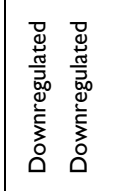 & 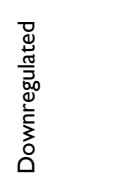 \\
\hline 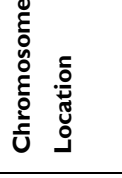 & $\frac{m}{\overline{\frac{1}{x}}}$ & 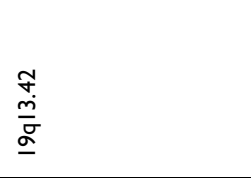 & \begin{tabular}{|l}
$\overline{\tilde{J}}$ \\
$\underline{-}$
\end{tabular} & 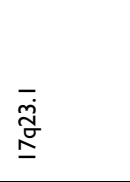 & $\frac{m}{\overline{\bar{x}}}$ & 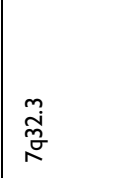 & $\frac{M}{\underline{m}}$ & 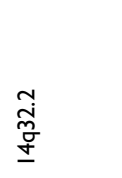 \\
\hline $\begin{array}{l}\frac{\mathbb{\alpha}}{\alpha} \\
\frac{\alpha}{\varepsilon}\end{array}$ & $\begin{array}{l}\overline{\tilde{T}} \\
\text { 产 } \\
\end{array}$ & 室 $\frac{\mathbb{D}}{n}$ & 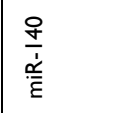 & 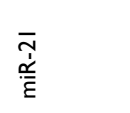 & 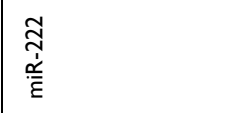 & 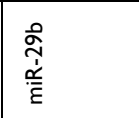 & 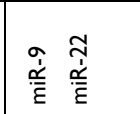 & 容害 \\
\hline 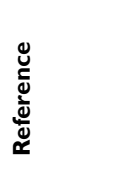 & 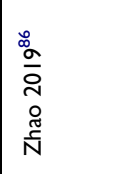 & 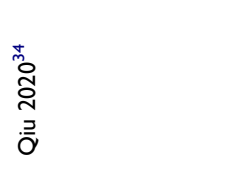 & 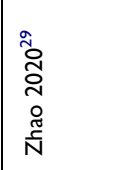 & 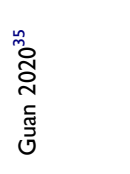 & 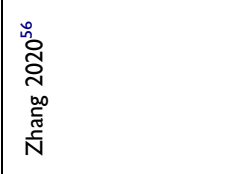 & 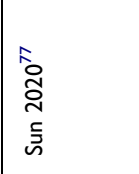 & 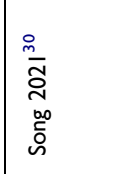 & 离 \\
\hline
\end{tabular}




\begin{tabular}{|c|c|c|c|}
\hline 1 & 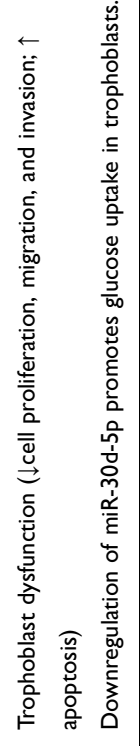 & 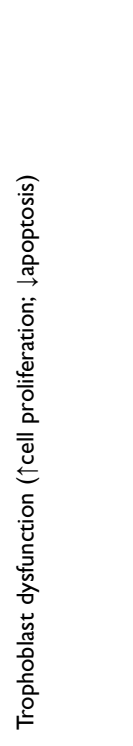 & 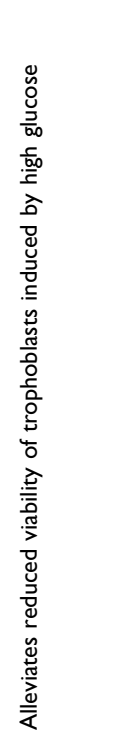 \\
\hline 1 & 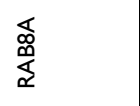 & 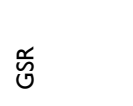 & 1 \\
\hline 1 & 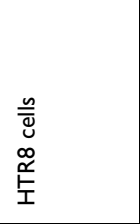 & 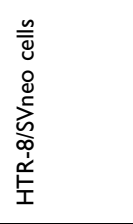 & 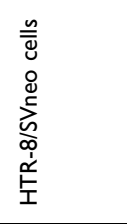 \\
\hline $\begin{array}{l}\text { 鵶 } \\
\text { 竞 }\end{array}$ & $\begin{array}{l}\text { 点 } \\
\text { 胥 }\end{array}$ & $\begin{array}{l}\text { 惡 } \\
\text { 空 }\end{array}$ & $\begin{array}{l}\text { 堊 } \\
\text { 竞 }\end{array}$ \\
\hline 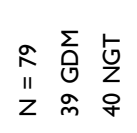 & 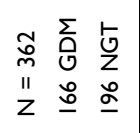 & $\begin{array}{lll} & 5 & 5 \\
\infty & 0 & 0 \\
11 & 0 & z \\
z & o & 9\end{array}$ & 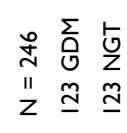 \\
\hline 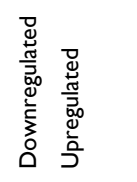 & 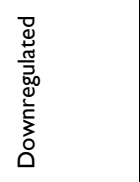 & 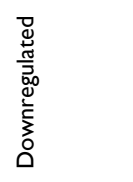 & 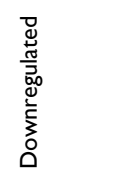 \\
\hline 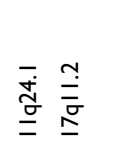 & 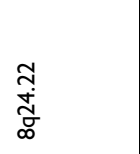 & $\frac{\stackrel{n}{\underline{\underline{n}}}}{\overline{\hat{x}}}$ & 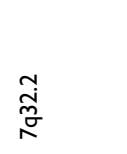 \\
\hline 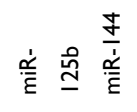 & 㸓 & 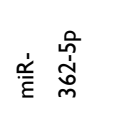 & 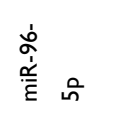 \\
\hline 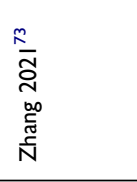 & 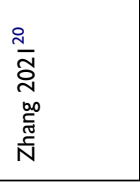 & 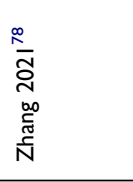 & $\frac{\sqrt{5}}{\stackrel{ \pm}{\Sigma}}$ \\
\hline
\end{tabular}

of their direct target genes, that were previously reported to participate in the GDM-associated pathogenesis.

\section{Placental Metabolic Imbalance}

The placenta promotes metabolic adaptations during pregnancy, augmenting maternal IR and regulating nutrient composition and supply from the mother to the fetus. ${ }^{27}$ It could be a "metabolic mirror," reflecting the health status of the mother and fetus. ${ }^{5,27}$ Therefore, it is essential to evaluate any placental metabolic imbalance and its underlying molecular mechanism in GDM.

The insulin signaling pathway components, including the insulin receptor, insulin receptor substrate 1 (IRS-1), insulin receptor substrate 2 (IRS-2), phosphatidylinositol 3-kinase (PI3K), glucose transporter 1 (GLUT1), and glucose transporter 4 (GLUT4), have been reported to be altered in placentas of GDM patients. Insulin sensitivity in patients with GDM was decreased by $40 \%$ compared with that in healthy controls, possibly owing to defective insulin signaling. ${ }^{28}$ Zhao et $\mathrm{al}^{29}$ found that miR-140-3p was overexpressed in placentas of GDM patients $(n=33)$ relative to healthy controls $(n=20)$, and related this effect to defective IR signaling by directly targeting the insulin receptor and the insulin-like growth factor 1 receptor (IGF-1R). A recent study demonstrated that miR-9 and miR-22 play a role in disordered glucose metabolism in the placentas of patients with GDM by targeting GLUT1 and hexokinase-2 (HK2). ${ }^{30}$ HK2 catalyzes the first step of most glucose metabolism pathways by phosphorylating glucose to produce glucose6-phosphate. The GLUT1 and HK2 protein levels were increased in patients with GDM $(\mathrm{n}=31)$ compared with those in healthy controls $(n=20)$, and miR-9 and miR-22 levels were reduced. GLUT1 was identified as a target for both miR-9 and miR-22, whereas HK2 was identified as an miR-9 target.

Specifically, more miRNAs, such as miR-137 and miR143 , were significantly dysregulated in pharmacologically treated patients than in diet-treated patients and healthy controls. Peng et $\mathrm{al}^{31}$ investigated the expression and role of miR-137 in placenta specimens from insulin-treated (A2GDM, $\mathrm{n}=11$ ) and lifestyle intervention-treated (A1GDM, $n=9$ ) women with GDM and healthy glucosetolerant women (NGT, $\mathrm{n}=20$ ). miR-137 expression was upregulated and that of its target gene, fibronectin type III domain-containing protein 5 (FNDC5), was downregulated in the placentas of insulin-treated patients with GDM. No significant difference was observed between the NGT and A1GDM women. FNDC5 is known as 
a key metabolic modulator involved in energy homeostasis and IR. Another study demonstrated that miR-143 expression was downregulated in patients with GDM receiving medication (A2GDM, $\mathrm{n}=6$ ) compared with that in diettreated patients with GDM (A2GDM, $\mathrm{n}=6$ ) and NGT women $(n=6)$; the levels of glycolytic enzymes, GLUT1, HK-2, phosphofructokinase (PFK), and lactate dehydrogenase (LDH) were significantly upregulated. A luciferase reporter assay confirmed that miR-143 directly regulated HK2 expression. ${ }^{32}$

Peroxisome proliferator-activated receptors (PPARs) are members of the steroid hormone superfamily. These include three subtypes (PPAR- $\alpha$, PPAR- $\beta / \delta$, and PPAR- $\gamma$ ) that are involved in lipid metabolism, inflammation, placental development, and angiogenesis. ${ }^{33}$ miR-518d and miR-21-5p were dysregulated in GDM placentas and both were negatively correlated with the protein levels of their predicted target gene, PPAR.$^{34-36}$ Notably, the trend of PPAR- $\alpha$ protein levels in GDM placental tissue compared with that in the controls was inconsistent in these two studies, possibly due to the sample sizes and different populations. miR-518d is a member of the placentaspecific miRNA cluster, C19MC, and is located on chromosome $19 \mathrm{q} 13.42 .{ }^{18}$ Zhao et $\mathrm{al}^{36}$ reported that miR-518d expression was specifically upregulated at 37-40 weeks of gestation in placentas of women with GDM $(n=40)$ relative to that in healthy controls $(n=40)$. In 2020, another study detected a consistent trend of miR-518d and PPAR- $\alpha$ expression in 120 placental samples (GDM: control $=60: 60) .{ }^{34}$ miR-21, located on chromosome 17q23.1, has attracted much attention as an important molecule related to tumors and diabetes. ${ }^{35}$ A more recent study demonstrated a negative correlation between placental miR-21-5p and PPAR- $\alpha$ expression in 295 patients $($ GDM:control $=137: 158) .{ }^{35}$ The authors found that the serum expression of miR-21 in patients with GDM and GDM rat models was lower than that in the controls, and that miR-21 expression in GDM rats decreased with an increase in blood glucose concentration, suggesting that miR-21 is an indicator of GDM development.

These data confirmed that miRNAs play a role in GDM placental metabolic dysfunction, potentially altering placental nutrient transport and fetal growth and programming the offspring for disease later in life.

\section{Oversized Placentas and Newborns}

Oversized placentas and newborns have been observed in women with GDM, especially in those with poor glycemic control. ${ }^{37}$ miRNAs may also play an important role in regulating placental and fetal growth during pregnancy. ${ }^{16}$ Ding et $\mathrm{al}^{23}$ revealed that the placental weight and placenta to baby weight ratio were significantly increased, and the incidence of large for gestational age babies was also elevated in GDM. To identify miRNAs involved in oversized placentas and newborns in GDM, the authors presented an integrated analysis of miRNA-mRNA expression in eight pairs of GDM and NGT placentas and validated the abnormal expression of five miRNAs in larger samples using qRT-PCR (GDM:NGT $=20: 18$ ). Bioinformatic analysis indicated that these aberrant miRNAs were enriched in "Cellular Development and Function, Organ Morphology, Organismal Development" and miR-138-5p was a central "node" in the miRNAmRNA network. Furthermore, the authors demonstrated that the downregulation of miR-138-5p in GDM placentas exerted a pathological role by targeting the transducin beta like $1 \mathrm{X}$-linked $(T B L 1 X)$ gene. TBL1X acts as an oncogene in $\mathrm{Wnt} / \beta$-catenin signaling, a pivotal pathway implicated in the human placenta and in IR regulation. ${ }^{38-40}$ Another study identified 29 dysregulated miRNAs using a miRNA array of placental tissue from five healthy controls and five patients with GDM. Additionally, 15 miRNAs whose targeted genes were involved in the epidermal growth factor receptor (EGFR)/PI3K/Akt pathway were identified using bioinformatics analysis. ${ }^{22}$ The EGFR/PI3K/Akt pathway plays an important role in placental development and fetal growth. ${ }^{41}$ Nine of the fifteen miRNAs were confirmed by qRT-PCR to be abnormally expressed in healthy $(n=10)$ and GDM $(n=10)$ placental tissue, of which miR-508-3p was the most upregulated. The authors observed that the EGFR, PI3K, and phospho-Akt protein levels were increased, and the level of PIKfyve (a FYVE finger-containing phosphoinositide kinase), a negative EGFR signaling regulator, was decreased in GDM tissues; they also demonstrated that miR-508-3p directly targeted PIKfyve. Thus, downregulated expression of the PI3K/ Akt-targeting miRNAs and upregulated expression of miR-508-3p, which suppresses PIKfyve, may play a role in the pathology of fetal overgrowth by EGFR/PI3K/Akt pathway activation.

\section{Inflammatory Response}

Placenta-derived inflammatory cytokines can stimulate immune cells and aggravate the immune/inflammatory response, thereby exacerbating chronic inflammation, increasing maternal IR, and inducing $\beta$ cell failure during 
pregnancy. ${ }^{42-50}$ Moreover, the immune cells and inflammatory cytokines are important parts of the placental microenvironment, which is essential for normal pregnancy. ${ }^{51-53}$ The imbalanced immune infiltration in the placenta participates in the pathogenesis and development of pregnancy-specific diseases, including GDM, and may affect GDM-related adverse pregnancy outcomes and clinical prognosis. ${ }^{49,52,54}$ The expression of miR-657 was found to be increased in placenta-derived mononuclear macrophages from patients with GDM $(n=48)$ compared with normal pregnancies $(n=46)$, along with the opposite trend of its target gene IL-37. ${ }^{26}$ IL-37, an antiinflammatory cytokine, can inhibit the activation of the nuclear factor kappa-B (NF- $\mathrm{B}$ ) signaling pathway and thus influence the production of inflammatory cytokines, such as IL- 6 and TNF- $\alpha .{ }^{55}$ As mentioned above, miR-518d may contribute to the inflammation in GDM through targeting PPAR- $\alpha$, along with increased levels of relevant inflammatory factors (NF- $\kappa$ B, TNF- $\alpha$, IL-1 $\beta$, IL-6, and cyclooxygenase-2 (COX-2)) in the placenta tissues and peripheral plasma of pregnant women with GDM. ${ }^{34}$ A more recent study demonstrated a negative correlation between placental miR-222 and its target gene C-X-C chemokine receptor type 4 (CXCR4) expression in 226 placental samples (GDM:control $=113: 113){ }^{56}$ CXCR4, the receptor of C-X-C ligand 12 (CXCL12), belongs to the subfamily of chemokine receptor $\mathrm{C}-\mathrm{X}-\mathrm{C}$ and is involved in various inflammation-related processes. $^{57}$ By post-transcriptionally regulating the inflammatory genes, miR-657, miR-518d, and miR-222 are possibly engaged in the inflammatory response associated with GDM.

\section{Placenta-Specific IncRNAs and circRNAs in GDM}

lncRNAs and circRNAs have gained attention as critical regulatory factors in human disease onset and progression, such as cancers and metabolic diseases; ${ }^{11,13}$ however, few studies have investigated the role of placenta-specific lncRNAs and circRNAs in GDM. Our preliminary study using microarray analysis to compare GDM with healthy control placentas identified 172 differentially expressed lncRNAs (86 up- and 86 down-regulated). ${ }^{21}$ Seven abnormally expressed IncRNAs were further validated by qRTPCR in an independent set of healthy $(n=20)$ and GDM $(\mathrm{n}=20)$ placenta samples. While lacking deep functional assays, the study focused on single lncRNAs.
Three studies have identified a link between dysregulated single lncRNAs in GDM placentas. ${ }^{25,63,68}$ The well-studied IncRNA, metastasis-associated lung adenocarcinoma transcript 1 (MALAT1), is a highly conserved lncRNA located on the short arm of human chromosome 11q13.1. ${ }^{5,59}$ Accumulated data reveal that MALAT1 plays a role in various diabetes-related complications by inducing pro-inflammatory cytokines and apoptosis in different cell types. ${ }^{60}$ Zhang et al reported that MALAT1 is expressed at higher levels in GDM placentas $(\mathrm{n}=78)$ compared to those of healthy controls $(\mathrm{n}=$ 38). ${ }^{25}$ The lncRNA plasmacytoma variant translocation 1 (PVT1), located on chromosome 8q24.21, is a common retroviral integration site in murine leukemia virus (MLV)-induced T lymphomas. ${ }^{61}$ It functions as an important oncogene owing to its facilitation of proliferation, invasion, and migration in tumor cells. $^{62}$ In regards to the significant similarities between the biological behavior of placental and cancer cells, a recent study identified the clinical significance and biological function of PVT1 in trophoblasts. The authors observed that PVT1 expression was attenuated in GDM placentas and in patients with preeclampsia. ${ }^{63}$ The lncRNA maternally expressed gene 3 (MEG3) appears to be an imprinted gene located on human chromosome $14 \mathrm{q} 32.2 .{ }^{64}$ Numerous studies have demonstrated that MEG3 possesses tumor suppressor activity and is involved in diabetes-related microangiopathy, IR, and pancreatic $\beta$-cell dysfunction. ${ }^{65-67}$ The blood and placental villous tissue in pregnant GDM patients $(n=20)$ showed increased MEG3 levels compared with those in non-GDM patients $(n=20) .{ }^{68}$

The recently discovered ncRNA, circRNA, plays an important role in various diseases, including atherosclerosis, diabetes, and cancer. ${ }^{11}$ However, few studies have explored the relationship between placental circRNAs and GDM. Wang et al $^{69}$ detected 46 differentially expressed circRNAs in GDM placentas using RNA-seq analysis and confirmed 10 of them using qRT-PCR. They also determined the association of differentially expressed circRNAs with GDM occurrence and development using bioinformatics analysis. Additionally, Yan et al reported 482 atypically expressed circRNAs in the placental villi of patients with GDM. ${ }^{24}$ In our previous study, we identified 114 differentially expressed circRNAs in GDM placentas and found that their expression profiles differed from those described above $^{21}$ possibly due to the use of different prediction tools, regions, populations, and database sequencing systems. Single placental circRNAs have rarely been reported in GDM. However, in 2020, Wang et al reported that hsa circ_0005243 expression was significantly downregulated in the placentas and plasma of GDM patients. $^{70}$ 
The biological functions of MALAT1, PVT1, MEG3, and hsa_circ_0005243 in GDM have been explored by cell experiments, which will be discussed in detail below.

\section{Diagnostic Role of Placenta-Specific ncRNAs in GDM}

In view of the co-expression in peripheral blood or circulating exosomes, several placenta-specific ncRNAs may serve as diagnostic markers in GDM, including miR-185, ${ }^{71} \mathrm{miR}-$ $132,{ }^{72}$ miR-518d, ${ }^{34}$ miR-140, ${ }^{29}$ miR-125b, ${ }^{73}$ miR- $144,{ }^{73}$ miR-96-5p, ${ }^{74}$ and lncRNA MEG3. ${ }^{67}$ Three studies have evaluated the diagnostic value of the placenta-specific ncRNAs. The area under the curves (AUCs) of miR-185 and miR-132 in diagnosing GDM were 0.927 and 0.898 , respectively. A model that combined miR-125b, miR-144 and body mass index before pregnancy showed an excellent diagnostic value with an AUC of 0.898. Early diagnosis is essential in reducing GDM-associated complications, and common screening of GDM depends on oral glucose tolerance test (OGTT) during 24-28 weeks of gestation. However, most studies obtained ncRNAs from blood sample during the third trimester and only one study focused on the placenta-derived exosomes obtained blood samples during the second trimester. Further research toward the potential diagnostic ability of placenta- specific ncRNAs in the second or even first trimester is still needed.

\section{Function of Placenta-Specific ncRNAs in GDM}

Identifying the molecular pathways and biological processes involved in GDM pathogenesis is essential for its prevention and may provide future therapeutic targets. Targeting a single ncRNA potentially affects multiple downstream pathways and several phenotypes, thus amplifying the effect of a single target and contributing to unexpected adverse effects. Therefore, the functions of ncRNAs should be completely understood both in vitro and in vivo before they are used for therapeutic purposes. Table 1 summarizes the biological function of placenta-specific miRNAs in GDM.

\section{Trophoblast Dysfunction}

Hormone secretion, vascularization, and the immunological interface of the placenta are directly related to several critical trophoblast cell functions, including proliferation, invasion, migration, and cell cycle progression. ${ }^{51}$ Moreover, a large amount of evidence indicates that the manipulation of ncRNA expression leads to functional alterations in trophoblast cells. Figure 1 summarizes and illustrates the role of
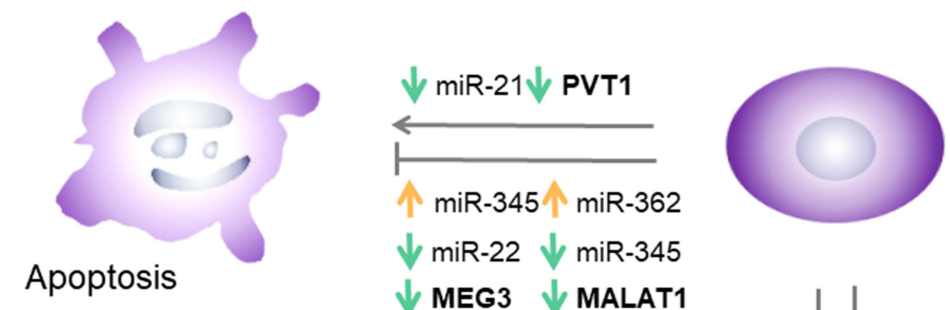

$\Downarrow$ MALAT1 $\downarrow$ PVT1

$\uparrow \operatorname{miR}-137 \downarrow$ miR-21

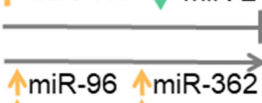

TmiR-132 miR-345

$\checkmark$ miR-138 $\downarrow$ miR-9

$\checkmark$ miR-22 $\downarrow$ miR-30d

$\checkmark$ MEG3 $\vee$ circ_0005243

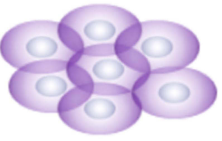

(n)

$\uparrow_{\operatorname{miR}-345} \mid \uparrow_{\operatorname{miR}-137}$

$\downarrow$ miR-138 $\downarrow$ miR-21

$\downarrow$ miR-29b $\quad \downarrow$ MALAT1

$\checkmark$ miR-30d $\quad \downarrow$ PVT1

$\downarrow$ MEG3 $\downarrow \perp \downarrow$ circ_0005243

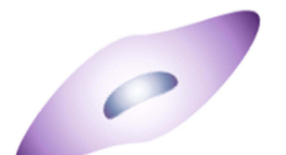

Invasion/

Migration

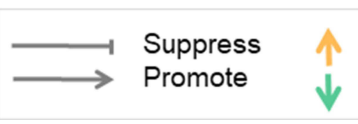

Overexpression

Knockdown

Figure I Effects of aberrantly expressed ncRNAs on trophoblast cells in GDM.

Notes: Green arrows indicate ncRNA knockdown. Orange arrows indicate ncRNA overexpression. Different from miRNAs, circRNAs and IncRNAs are in bold italics and bold, respectively. Black arrows indicate a promotive effect on trophoblast function. Black T-bars indicate an inhibitory effect on trophoblast function.

Abbreviations: GDM, gestational diabetes mellitus; ncRNA, non-coding RNA; miR, microRNA; MEG3, maternally expressed gene 3; PVTI, plasmacytoma variant translocation I; MALATI, metastasis-associated lung adenocarcinoma transcript I; circ_0005243, hsa_circ_0005243 (a circular RNA). 
ncRNAs in trophoblast dysfunction and the phenotypes obtained when altering the expression of single ncRNAs.

The altered expression of placental miRNAs, including miR-21, miR-132, miR-137, miR-9, miR-22, miR-29b, miR-345, miR-138, miR-30d, miR-362, miR-96, and miR193, in GDM can cause disordered biological behavior and abnormal cell cycle progression of trophoblasts. HTR-8/ SVneo, a cell line derived from first-trimester extravillous cytotrophoblast cells, is often used for in vitro experiments on placental dysfunction. ${ }^{75}$ The miR-21 mimic transfected into HTR8/SVneo cells promoted cell proliferation, migration, and infiltration and inhibited apoptosis; the opposite effects were observed in the miR-21 inhibitor group. ${ }^{35}$ The overexpression of miR-137 and miR-138 suppressed the viability and migration of HTR-8/SVneo cells, whereas miR-138 knockdown promoted cell migration and proliferation. ${ }^{23,31}$ Inhibitors of miR-9 and miR-22 enhanced viability and prevented apoptosis in primary syncytiotrophoblasts (STBs) and HTR-8/SVneo cells, which could be partially rescued by silencing GLUT1 and HK2. ${ }^{30}$

Additionally, Li et al $^{19}$ revealed that miR-345-3p expression decreased in GDM placentas and maternal blood $(n=30)$ compared with that in healthy controls $(n=30)$. Functional assays have shown that miR-345-3p overexpression could inhibit apoptosis and promote cell proliferation and migration in trophoblasts by targeting B-cell lymphoma 2 antagonist/killer 1 (BAK1), a member of the B-cell lymphoma 2 protein family involved in mitochondrial apoptosis. $^{76}$

miR-29b and miR-30d-5p levels were downregulated in terminal placentas of patients with GDM relative to those in healthy controls; the overexpression of miR-29b and miR-30d-5p contributed to reduced cell proliferation, migration, and invasion and increased apoptosis in HTR-8/ SVneo cells. ${ }^{20,77}$ Dual-luciferase reporter system analysis showed that miR-29b was inversely regulated by hypoxiainducible factor 3-alpha $(H I F 3 A)$, a gene associated with apoptosis and proliferation. The restoration of HIF3A partially antagonized the inhibitory effect of miR-29b overexpression on cell growth and migration, and the downregulation of HIF3A restricted the enhanced cell migration induced by miR-29b knockdown. Functional assays showed that Ras-related protein Rab-8A (RAB8A) is the direct target of miR-30d-5p and the downregulation of RAB8A could attenuate the increase in trophoblast cell proliferation, migration, invasion and glucose uptake in trophoblast cells induced by the miR-30d-5p inhibitor.
RAB8A, a key regulator of intracellular membrane trafficking, is involved in glucose homeostasis and autophagy.

Furthermore, the activities of miR-132, miR-362-5p and miR-96-5p may emerge as protection against GDM through the mediation of trophoblast cell function in high glucose $(\mathrm{HG})$. Zhou et $\mathrm{al}^{72}$ revealed decreased miR-132 expression in serum and placenta tissues in GDM patients compared with healthy women. In vitro studies showed that HG treatment induced the inhibition of HTR-8/SVneo cell proliferation and the expression of miR-132, and the HG-induced suppressed cell proliferation can be substantially rescued by overexpressing miR-132. Another study demonstrated the reduced expression of miR-362-5p in HG-treated HTR-8/SVneo cells and placental tissues of patients with GDM; the downregulation of miR-362-5p expression was negatively correlated with the expression of its predicted target gene, glutathione-disulfide reductase (GSR). The study revealed that miR-362-5p promoted trophoblast proliferation and inhibited apoptosis via the targeting of GSR and the activation of PI3K/AKT pathway. ${ }^{78}$ A more recent study revealed that miR-96-5p levels were significantly decreased in the placenta and plasma samples of patients with GDM as well as in HGtreated HTR-8/SVneo cells. Moreover, the overexpression of miR-96-5p could alleviate high glucose-induced reduction in the cell viability of trophoblasts. ${ }^{74}$

Evidence for the involvement of dysregulated placental lncRNAs and circRNAs in trophoblast dysfunction in GDM is rather poor. Cell experiments showed that MALAT1 attenuated the proliferation, invasion, and migration of HTR-8 cells, possibly via the $\mathrm{TGF}-\beta / \mathrm{NF}-\kappa \mathrm{B}$ signaling pathway. However, the mechanism by which it modulates the TGF$\beta / \mathrm{NF}-\kappa \mathrm{B}$ signaling pathway is unclear. $^{25}$ Suppression of PVT1 expression enhanced apoptosis and inhibited the proliferation, migration, and invasion of trophoblast cells. Moreover, significantly decreased Akt phosphorylation and the aberrant expression of $C C N D 1, G D P D 3, I T G A V, I T G B 8$, $P D G F B$, and EIF4EBP1 genes enriched in the PI3K/Akt pathway were observed after PVT1 knockdown, suggesting that PVT1 functions through the PI3K/Akt pathway. ${ }^{63}$ Subcellular localization reflects lncRNA function to some extent. lncRNAs located in the nucleus mainly regulate transcription, alternative splicing, or modified chromatin structures; those in the cytoplasm can influence protein expression by sponging miRNA away from their coding gene targets. ${ }^{13}$ PVT1 was mainly distributed in the nucleus and cytoplasm of trophoblast cells, indicating its diversified biological functions. IncRNAs can act as ceRNA molecules or efficient 
miRNA sponges to regulate downstream gene expression. miR-345-3p has been identified as a direct target of MEG3, the expression of which was downregulated in GDM. In vitro studies have demonstrated that MEG3 can attenuate cell viability, migration, and invasion and promote apoptosis by sponging miR-345-3p. ${ }^{68}$ As for circRNA, silencing hsa_circ_0005243 significantly suppresses cell proliferation and migration abilities of trophoblast cells. Reduced $\beta$-catenin expression and increased NF- $\mathrm{kB}$ p65 nuclear translocation were observed after hsa_circ_0005243 knockdown. ${ }^{70}$ Thus, the downregulation of hsa_circ_0005243 expression plays a role in GDM pathogenesis by inducing trophoblast cell dysfunction via the $\beta$-catenin and NF- $\mathrm{KB}$ signaling pathways; however, the underlying mechanism is unclear.

\section{Intracellular Glucose Metabolism}

Placental glucose metabolism interacts reciprocally with other carbon substrates, which contributes to nutrient transfer, energy balance, fetal growth, and hence, fetal programming. It is known that glucose transport in the placenta is controlled by extracellular glucose concentration, GLUTs expression in the basal membrane, and the intracellular glucose metabolism. ${ }^{79}$ Hyperglycemia during GDM results in changes in glucose transport in the placenta. Therefore, assessing intracellular glucose metabolism is essential to better understand the placental glucose metabolism in GDM.

In vitro experiments indicated that miR-9 and miR-22 inhibitors increased glucose uptake and lactate secretion in primary syncytiotrophoblasts (STBs) and HTR8/SVneo cells by targeting GLUT1 and HK2. ${ }^{30}$ Another study revealed that downregulation of miR-30d-5p promoted glucose uptake of HTR8 cells, which could be attenuated by downregulation of RAB8A. However, there were no differences between the miR-30d-5p mimic and pre-miR control on cell glucose uptake of HTR8 cells. ${ }^{20}$ Since IR signaling regulates the glucose uptake of placental cells, Zhao et al demonstrated that miR-140 repressed the expression of IR- $\alpha$ and IGF1R on a post-transcriptional level in HTR8 /SVneo cells. The authors also confirm that miR-140 inhibits IR- $\alpha$ and IGF1R through targeting 3'UTRs in HEK293 cells. Moreover, the glucose uptake in both HTR-8/SVneo and HUVEC cells was repressed by the miR-140 mimic and upregulated by miR-140 inhibitor. $^{29}$

Physiologically, at least $30 \%$ of glucose entering the placenta is metabolized by glycolysis or aerobic glycolysis through mitochondrial oxidative phosphorylation to generate energy. ${ }^{80}$ Muralimanoharan et al reported a reduction in mitochondrial respiration by half in trophoblast cells isolated from A2GDM placentas. The overexpression of miR-143 contributed to increased mitochondrial respiration and protein expression of mitochondrial complexes, and decreased expression of glycolytic enzymes by $40 \%$. Downregulation of miR-143 partially rescued mitochondrial function and reduced levels of glycolytic enzymes in trophoblast cells isolated from A2GDM placenta. $^{32}$

\section{Pro-Inflammatory Effect}

The altered levels of inflammatory cytokines and dysregulation of immune cells are initiating factors of GDM, which aggravate the chronic inflammatory reaction and the degree of maternal IR. ${ }^{54,81}$ For instance, placental macrophages switch to a pro-inflammatory phenotype in pregnancies with diabetes, along with an elevated expression of pro-inflammatory cytokines in the placenta. ${ }^{82,83}$ Emerging evidence has supported the critical roles of ncRNAs in the inflammatory response and the development of metabolic diseases, while limited studies have investigated the pro-inflammatory role of placentaspecific ncRNAs in GDM.

As described above, Wang et al demonstrated the upregulation of miR-657 in placenta-derived mononuclear macrophages from patients with GDM compared with normal pregnancies. In cell experiments, the authors revealed that miR-657 could promote the proliferation of mononuclear macrophages, the generation of inflammatory cytokines (IL-6 and TNF- $\alpha$ ) and activation of NF- $\kappa$ B in lipopolysaccharide-induced mononuclear macrophages by targeting IL-37. ${ }^{26}$ The NF- $\mathrm{kB}$ signaling pathway has been demonstrated to regulate the cytokine network and is closely correlated with GDM pathogenesis. ${ }^{84}$ miR-518d, lncRNA MALAT1 and hsa_circ_0005243 also mediate the inflammatory response through NF- $\mathrm{KB}$ signaling. As mentioned above, miR-518d was upregulated in the placentas of patients with GDM, along with the relatively high inflammatory responses both in the placenta tissues and peripheral plasma. In vitro experiments demonstrated that miR-518d was associated with the inflammatory responses through the PPAR $\alpha$-mediated NF- $\kappa B$ Pathway in HTR8/SVneo. ${ }^{34}$ hsa_circ_0005243 was significantly downregulated in both the placenta and plasma of GDM patients. In vitro, the depletion of hsa_circ_0005243 significantly increased the secretion of the pro-inflammatory factors TNF- $\alpha$ and IL- 6 in trophoblast cells, as well as 
reduced $\beta$-catenin expression and increased NF- $\kappa B$ p65 nuclear translocation. ${ }^{70}$ Higher lncRNA MALAT1 expression was observed in GDM placental tissues compared to a normal control group. Cell experiments showed that MALAT1 knockdown diminished the secretion of proinflammatory factors (TNF- $\alpha$ and IL-6) and suppressed the expression of TGF- $\beta$ and NF- $\kappa B .{ }^{25}$ Accordingly, dysregulation of miR-657, miR-518d, lncRNA MALAT1 and has_circ_0005243 may contribute to the pathogenesis of GDM by activating the inflammatory response via NF- $\kappa B$ signaling.

Apart from in vitro studies, more recent research highlighted the pro-inflammatory role of placenta-specific miRNAs in GDM in vivo. Zhang et al established a GDM mouse model by intraperitoneal injection of streptozocin (STZ); based on this model, they found that miR222 was increased while the expression of its target gene CXCR4 was decreased in the placenta and pancreatic tissues of GDM mice, consistent with previous results in human placentas. In vivo, silencing miR-222 could diminish the inflammatory response in GDM mice through CXCR4 enhancement and NLRP3 inflammasome inactivation. ${ }^{56}$ In another study, the mouse model of GDM was successfully established by combination of high sugar and high fat feeding and injecting STZ after 15 days feeding; the blocked nuclear transport process of $\mathrm{NF}-\mathrm{\kappa B}$ and the weakened phosphorylation of NF-KB pathway-related proteins were observed after administrating the miR-518d antagomir, which could be reversely regulated by inhibiting PPAR $\alpha$. These results agreed with that in the in vitro study mentioned above and further proved that miR-518d promotes the process of NF- $\kappa B$ nuclear transport to regulate the inflammatory responses through negatively regulating PPAR $\alpha .{ }^{34}$

Notably, other studies also reported placenta-specific miRNAs, such as miR-98 and miR-221, were dysregulated and associated with the pathogenic processes of GDM. miR-98 is elevated in the placenta of GDM patients and it is linked to global DNA methylation via targeting of methyl $\mathrm{CpG}$ binding protein 2 (Mecp2). ${ }^{85}$ Cao et al revealed that miRNA-221 was downregulated in the placentas of GDM rats induced by high sugar and high fat diet, and negatively correlated to the glycemic level. In in vitro experiments, the depletion of miRNA-221 suppressed insulin secretion, proliferation and stimulated apoptosis in islet $\beta$ cells by targeting PAK1 $1{ }^{86}$ However, it seems incomplete to study the role of placenta-specific molecules directly on islet cells without verifying their expression in peripheral blood or the pancreas.

\section{Conclusion and Prospects}

GDM is an increasingly common complication in pregnancy that acts as a threat to the short- and long-term health of the mother and her offspring. Placental dysfunction is a unique pathophysiological feature of GDM that is closely correlated with its development and complications. A growing number of ncRNAs are being identified in GDM placentas, highlighting their potential involvement in the pathogenic mechanisms of pregnancy-related complications. The literature described herein provide strong evidence that placenta-specific ncRNAs play important roles in GDM, such as effects on placental structure, function and metabolism, effects on trophoblast dysfunction, intracellular glucose metabolism and inflammation. So far, there are numerous studies on the underlying molecular mechanism of action of differentially expressed ncRNAs, while few focused on IncRNAs and circRNAs in GDM placentas. More dysregulated placenta-specific ncRNAs in GDM should be identified and explored both in quantity and diversity, especially in regards to the roles of circRNAs and lncRNAs. Due to the transcriptional and post-transcriptional regulation of circRNAs and lncRNAs, more research should focus on the abnormal expression and functions of these specific IncRNAs or circRNAs, which may shed new light into the molecular mechanisms governing GDM. Considering the temporal manner of placenta-specific ncRNAs, evaluating their expression and exploring their functions during different trimesters until delivery in vivo and in vitro is essential to ascertain how they regulate placental development and trophoblast cell activities. Studies concerning crosstalk of placentalspecific ncRNAs, especially in exosomes, among different tissues in GDM will contribute to the full understanding of the local and systemic pathogenic mechanism of GDM. Notably, the multiple downstream phenotypes observed by manipulating the expression of single ncRNAs are due to their involvement in diverse molecular regulatory networks. Multiple ncRNAs are simultaneously implicated in regulation and downstream effects. To evaluate the therapeutic possibility of placenta-specific ncRNAs, more in vivo research in GDM animal models should be carried out. Further studies are needed to elucidate the mechanisms involved in the abovementioned events and explore the hitherto unidentified placenta-specific ncRNAs 
involved in GDM, in order to aid in the development of new or improved therapeutic options.

\section{Acknowledgment}

We gratefully acknowledge Yi Zhang for providing intellectual support and technical assistance.

\section{Funding}

The review was supported by the Clinical Research Project of the Liaoning Diabetes Medical Nutrition Prevention Society, Liaoning Province, People's Republic of China (grant number LNSTNBYXYYFZXH-RS01B). The sponsor is one of the authors of this review.

\section{Disclosure}

The authors report no conflicts of interest in this work.

\section{References}

1. Jovanovic L, Pettitt DJ. Gestational diabetes mellitus. JAMA. 2001;286(20):2516-2518. doi:10.1001/jama.286.20.2516

2. Cho NH, Shaw JE, Karuranga S, et al. IDF diabetes atlas: global estimates of diabetes prevalence for 2017 and projections for 2045 . Diabetes Res Clin Pract. 2018;138:271-281. doi:10.1016/j. diabres.2018.02.023

3. Lowe WL Jr., Scholtens DM, Kuang A, et al. Hyperglycemia and Adverse Pregnancy Outcome Follow-up Study (HAPO FUS): maternal gestational diabetes mellitus and childhood glucose metabolism. Diabetes Care. 2019;42(3):372-380. doi:10.2337/dc18-1646

4. Maryns AS, Dehaene I, Page G. Maternal and neonatal outcomes in a treated versus non- treated cohort of women with gestational diabetes mellitus according to the HAPO 5 and 4 criteria. Facts Views Vis Obgyn. 2017;9(3):133-140.

5. Gauster M, Desoye G, Totsch M, Hiden U. The placenta and gestational diabetes mellitus. Curr Diab Rep. 2012;12(1):16-23. doi:10.1007/s11892-011-0244-5

6. Kampmann U, Knorr S, Fuglsang J, Ovesen P. Determinants of maternal insulin resistance during pregnancy: an updated overview. J Diabetes Res. 2019;2019:5320156. doi:10.1155/2019/5320156

7. Fuglsang J, Sandager P, Moller N, Fisker S, Orskov H, Ovesen P. Kinetics and secretion of placental growth hormone around parturition. Eur J Endocrinol. 2006;154(3):449-457. doi:10.1530/ eje.1.02109

8. Lonberg U, Damm P, Andersson AM, et al. Increase in maternal placental growth hormone during pregnancy and disappearance during parturition in normal and growth hormone-deficient pregnancies. Am J Obstet Gynecol. 2003;188(1):247-251. doi:10.1067/ mob.2003.82

9. Roeder HA, Moore TR, Ramos GA. Changes in postpartum insulin requirements for patients with well-controlled type 1 diabetes. $\mathrm{Am}$ $J$ Perinatol. 2016;33(7):683-687. doi:10.1055/s-0036-1571323

10. Carrasco-Wong I, Moller A, Giachini FR, et al. Placental structure in gestational diabetes mellitus. Biochim Biophys Acta Mol Basis Dis. 2020;1866(2):165535. doi:10.1016/j.bbadis.2019.165535

11. Beermann J, Piccoli MT, Viereck J, Thum T. Non-coding RNAs in development and disease: background, mechanisms, and therapeutic approaches. Physiol Rev. 2016;96(4):1297-1325. doi:10.1152/ physrev.00041.2015

12. Ha M, Kim VN. Regulation of microRNA biogenesis. Nat Rev Mol Cell Biol. 2014;15(8):509-524.
13. Tsagakis I, Douka K, Birds I, Aspden JL. Long non-coding RNAs in development and disease: conservation to mechanisms. J Pathol. 2020;250(5):480-495. doi:10.1002/path.5405

14. Kristensen LS, Andersen MS, Stagsted LVW, Ebbesen KK, Hansen TB, Kjems J. The biogenesis, biology and characterization of circular RNAs. Nat Rev Genet. 2019;20(11):675-691. doi:10.1038/ s41576-019-0158-7

15. Khalifa O, Errafii K, Al-Akl NS, Arredouani A. Noncoding RNAs in nonalcoholic fatty liver disease: potential diagnosis and prognosis biomarkers. Dis Markers. 2020;2020:8822859. doi:10.1155/2020/ 8822859

16. Fu G, Brkic J, Hayder H, Peng C. MicroRNAs in human placental development and pregnancy complications. Int J Mol Sci. 2013;14 (3):5519-5544. doi:10.3390/ijms14035519

17. Morales-Prieto DM, Chaiwangyen W, Ospina-Prieto S, et al. MicroRNA expression profiles of trophoblastic cells. Placenta. 2012;33(9):725-734. doi:10.1016/j.placenta.2012.05.009

18. Donker RB, Mouillet JF, Chu T, et al. The expression profile of C19MC microRNAs in primary human trophoblast cells and exosomes. Mol Hum Reprod. 2012;18(8):417-424. doi:10.1093/ molehr/gas013

19. Li Y, Zhuang J. miR-345-3p serves a protective role during gestational diabetes mellitus by targeting BAK1. Exp Ther Med. 2021;21 (1):2.

20. Zhang L, Li K, Tian S, et al. Down-regulation of microRNA-30d-5p is associated with gestational diabetes mellitus by targeting RAB8A. $J$ Diabetes Complications. 2021;35(8):107959. doi:10.1016/j. jdiacomp.2021.107959

21. Tang L, Li P, Li L. Whole transcriptome expression profiles in placenta samples from women with gestational diabetes mellitus. $J$ Diabetes Investig. 2020;11(5):1307-1317. doi:10.1111/ jdi. 13250

22. Li J, Song L, Zhou L, et al. A microRNA signature in gestational diabetes mellitus associated with risk of macrosomia. Cell Physiol Biochem. 2015;37(1):243-252. doi:10.1159/000430349

23. Ding R, Guo F, Zhang Y, et al. Integrated transcriptome sequencing analysis reveals role of miR-138-5p/ TBL1X in placenta from gestational diabetes mellitus. Cell Physiol Biochem. 2018;51(2):630-646. doi:10.1159/000495319

24. Yan L, Feng J, Cheng F, et al. Circular RNA expression profiles in placental villi from women with gestational diabetes mellitus. Biochem Biophys Res Commun. 2018;498(4):743-750. doi:10.1016/ j.bbrc.2018.03.051

25. Zhang Y, Qu L, Ni H, et al. Expression and function of lncRNA MALAT1 in gestational diabetes mellitus. Adv Clin Exp Med. 2020;29(8):903-910. doi:10.17219/acem/121524

26. Wang P, Wang H, Li C, et al. Dysregulation of microRNA-657 influences inflammatory response via targeting interleukin-37 in gestational diabetes mellitus. $J$ Cell Physiol. 2019;234 (5):7141-7148. doi:10.1002/jcp.27468

27. Brett KE, Ferraro ZM, Yockell-Lelievre J, Gruslin A, Adamo KB. Maternal-fetal nutrient transport in pregnancy pathologies: the role of the placenta. Int J Mol Sci. 2014;15(9):16153-16185. doi:10.3390/ ijms150916153

28. Ryan EA, Enns L. Role of gestational hormones in the induction of insulin resistance. J Clin Endocrinol Metab. 1988;67(2):341-347. doi:10.1210/jcem-67-2-341

29. Zhao C, Zhao C, Zhao H. Defective insulin receptor signaling in patients with gestational diabetes is related to dysregulated miR-140 which can be improved by naringenin. Int J Biochem Cell Biol. 2020;128:105824. doi:10.1016/j.biocel.2020.105824

30. Song TR, Su GD, Chi YL, Wu T, Xu Y, Chen CC. Dysregulated miRNAs contribute to altered placental glucose metabolism in patients with gestational diabetes via targeting GLUT1 and HK2. Placenta. 2021;105:14-22. doi:10.1016/j.placenta.2021.01.015 
31. Peng HY, Li MQ, Li HP. MiR-137 restricts the viability and migration of HTR-8/SVneo cells by downregulating FNDC5 in gestational diabetes mellitus. Curr Mol Med. 2019;19(7):494-505. doi:10.2174/ 1566524019666190520100422

32. Muralimanoharan S, Maloyan A, Myatt L. Mitochondrial function and glucose metabolism in the placenta with gestational diabetes mellitus: role of miR-143. Clin Sci. 2016;130(11):931-941. doi:10.1042/CS20160076

33. Grygiel-Gorniak B. Peroxisome proliferator-activated receptors and their ligands: nutritional and clinical implications-a review. Nutr J. 2014;13(1):17. doi:10.1186/1475-2891-13-17

34. Qiu H, Liu X, Yao S, Zhou J, Zhang X, Du J. Regulation and mechanism of miR-518d through the PPARalpha-mediated NF-kappaB pathway in the development of gestational diabetes mellitus. $J$ Diabetes Res. 2020;2020:7019597. doi:10.1155/2020/7019597

35. Guan CY, Tian S, Cao JL, Wang XQ, Ma X, Xia HF. Down-regulated miR-21 in gestational diabetes mellitus placenta induces PPAR-alpha to inhibit cell proliferation and infiltration. Diabetes Metab Syndr Obes. 2020;13:3009-3034. doi:10.2147/DMSO.S253920

36. Zhao C, Zhang T, Shi Z, Ding H, Ling X. MicroRNA-518d regulates PPARalpha protein expression in the placentas of females with gestational diabetes mellitus. Mol Med Rep. 2014;9(6):2085-2090. doi: $10.3892 / \mathrm{mmr} .2014 .2058$

37. Wu HH. [Enlightenment coming from HAPO study to HAPO follow-up study]. Zhonghua Yi Xue Za Zhi. 2019;99(34):2646-2649. [Chinese]

38. Zeng XC, Liu FQ, Yan R, et al. Downregulation of miR-610 promotes proliferation and tumorigenicity and activates Wnt/beta-catenin signaling in human hepatocellular carcinoma. Mol Cancer. 2014;13(1):261. doi:10.1186/1476-4598-13-261

39. Daniele G, Winnier D, Mari A, et al. Sclerostin and insulin resistance in prediabetes: evidence of a cross talk between bone and glucose metabolism. Diabetes Care. 2015;38(8):1509-1517. doi:10.2337/ dc14-2989

40. Knofler M, Pollheimer J. Human placental trophoblast invasion and differentiation: a particular focus on Wnt signaling. Front Genet. 2013;4:190. doi:10.3389/fgene.2013.00190

41. Forbes K, Westwood M. Maternal growth factor regulation of human placental development and fetal growth. J Endocrinol. 2010;207 (1):1-16. doi:10.1677/JOE-10-0174

42. Zhang J, Chi H, Xiao H, et al. Interleukin 6 (IL-6) and Tumor Necrosis Factor alpha (TNF-alpha) Single Nucleotide Polymorphisms (SNPs), inflammation and metabolism in gestational diabetes mellitus in Inner Mongolia. Med Sci Monit. 2017;23:4149-4157. doi:10.12659/MSM.903565

43. Li J, Li Y, Zhou X, et al. Upregulation of IL-15 in the placenta alters trophoblasts behavior contributing to gestational diabetes mellitus. Cell Biosci. 2021;11(1):33. doi:10.1186/s13578-02100533-4

44. Amirian A, Mahani MB, Abdi F. Role of interleukin-6 (IL-6) in predicting gestational diabetes mellitus. Obstet Gynecol Sci. 2020;63(4):407-416. doi:10.5468/ogs.20020

45. Schulze F, Wehner J, Kratschmar DV, et al. Inhibition of IL-1beta improves glycaemia in a mouse model for gestational diabetes. $\mathrm{Sci}$ Rep. 2020;10(1):3035. doi:10.1038/s41598-020-59701-0

46. Wei Q, Chen X, Chen H. Association of single nucleotide polymorphisms of the IL-6, IL-10, and TNF-alpha genes with susceptibility to gestational diabetes mellitus. Genet Test Mol Biomarkers. 2020;24(7):390-398. doi:10.1089/gtmb.2020.0069

47. Piao C, Wang X, Peng S, et al. IL-34 causes inflammation and beta cell apoptosis and dysfunction in gestational diabetes mellitus. Endocr Connect. 2019;8(11):1503-1512. doi:10.1530/ EC-19-0436

48. Yu Z, Liu J, Zhang R, et al. IL-37 and 38 signalling in gestational diabetes. J Reprod Immunol. 2017;124:8-14. doi:10.1016/j.jri.2017.09.011
49. Schumacher A, Sharkey DJ, Robertson SA, Zenclussen AC. Immune cells at the fetomaternal interface: how the microenvironment modulates immune cells to foster fetal development. J Immunol. 2018;201 (2):325-334. doi:10.4049/jimmunol.1800058

50. Yilmaz O, Kucuk M, Ilgin A, Dagdelen M. Assessment of insulin sensitivity/resistance and their relations with leptin concentrations and anthropometric measures in a pregnant population with and without gestational diabetes mellitus. $J$ Diabetes Complications. 2010;24(2):109-114. doi:10.1016/j.jdiacomp.2009.01.006

51. Knofler M, Haider S, Saleh L, Pollheimer J, Gamage T, James J. Human placenta and trophoblast development: key molecular mechanisms and model systems. Cell Mol Life Sci. 2019;76 (18):3479-3496. doi:10.1007/s00018-019-03104-6

52. Tong M, Abrahams VM. Immunology of the placenta. Obstet Gynecol Clin North Am. 2020;47(1):49-63. doi:10.1016/j. ogc.2019.10.006

53. Liu S, Diao L, Huang C, Li Y, Zeng Y, Kwak-Kim JYH. The role of decidual immune cells on human pregnancy. J Reprod Immunol. 2017;124:44-53. doi:10.1016/j.jri.2017.10.045

54. De Luccia TPB, Pendeloski KPT, Ono E, et al. Unveiling the pathophysiology of gestational diabetes: studies on local and peripheral immune cells. Scand J Immunol. 2020;91(4):e12860. doi:10.1111/ sji. 12860

55. Wang X, Xu K, Chen S, Li Y, Li M. Role of interleukin-37 in inflammatory and autoimmune diseases. Iran J Immunol. 2018;15 (3):165-174.

56. Zhang $\mathrm{H}$, Luan S, Xiao X, Lin L, Zhao X, Liu X. Silenced microRNA-222 suppresses inflammatory response in gestational diabetes mellitus mice by promoting CXCR4. Life Sci. 2021;266:118850. doi:10.1016/j.lfs.2020.118850

57. Kircher M, Herhaus P, Schottelius M, et al. CXCR4-directed theranostics in oncology and inflammation. Ann Nucl Med. 2018;32 (8):503-511. doi:10.1007/s12149-018-1290-8

58. Chaleshi V, Asadzadeh Aghdaei H, Nourian M, et al. Association of MALAT1 expression in gastric carcinoma and the significance of its clinicopathologic features in an Iranian patient. Gastroenterol Hepatol Bed Bench. 2021;14(2):108-114.

59. Onoguchi-Mizutani R, Kirikae Y, Ogura Y, Gutschner T, Diederichs S, Akimitsu N. Identification of a heat-inducible novel nuclear body containing the long noncoding RNA MALAT1. $J$ Cell Sci. 2021;134(10):10. doi:10.1242/jcs.253559

60. Abdulle LE, Hao JL, Pant OP, et al. MALAT1 as a diagnostic and therapeutic target in diabetes-related complications: a promising long-noncoding RNA. Int $J$ Med Sci. 2019;16(4):548-555. doi:10.7150/ijms.30097

61. Onagoruwa OT, Pal G, Ochu C, Ogunwobi OO. Oncogenic role of PVT1 and therapeutic implications. Front Oncol. 2020;10:17. doi:10.3389/fonc.2020.00017

62. Lu D, Luo P, Wang Q, Ye Y, Wang B. lncRNA PVT1 in cancer: a review and meta-analysis. Clin Chim Acta. 2017;474:1-7. doi:10.1016/j.cca.2017.08.038

63. Wang Q, Lu X, Li C, et al. Down-regulated long non-coding RNA PVT1 contributes to gestational diabetes mellitus and preeclampsia via regulation of human trophoblast cells. Biomed Pharmacother. 2019;120:109501. doi:10.1016/j.biopha.2019.109501

64. Ghafouri-Fard S, Taheri M. Maternally expressed gene 3 (MEG3): a tumor suppressor long non coding RNA. Biomed Pharmacother. 2019;118:109129. doi:10.1016/j.biopha.2019.109129

65. Wang N, Zhu Y, Xie M, et al. Long noncoding RNA meg3 regulates mafa expression in mouse beta cells by inactivating $\operatorname{Rad} 21, \mathrm{Smc} 3$ or Sin3alpha. Cell Physiol Biochem. 2018;45(5):2031-2043. doi:10.1159/000487983

66. Wei GH, Wang X. lncRNA MEG3 inhibit proliferation and metastasis of gastric cancer via p53 signaling pathway. Eur Rev Med Pharmacol Sci. 2017;21(17):3850-3856. 
67. Zhu X, Wu YB, Zhou J, Kang DM. Upregulation of lncRNA MEG3 promotes hepatic insulin resistance via increasing FoxO1 expression. Biochem Biophys Res Commun. 2016;469(2):319-325. doi:10.1016/j. bbrc. 2015.11 .048

68. Zhang H. Mechanism associated with aberrant IncRNA MEG3 expression in gestational diabetes mellitus. Exp Ther Med. 2019;18 (5):3699-3706.

69. Wang H, She G, Zhou W, Liu K, Miao J, Yu B. Expression profile of circular RNAs in placentas of women with gestational diabetes mellitus. Endocr J. 2019;66(5):431-441. doi:10.1507/endocrj.EJ18-0291

70. Wang H, Zhou W, She G, Yu B, Sun L. Downregulation of hsa_circ_0005243 induces trophoblast cell dysfunction and inflammation via the beta-catenin and NF-kappaB pathways. Reprod Biol Endocrinol. 2020;18(1):51. doi:10.1186/s12958-020-00612-0

71. Qi S, Wang X. Decreased expression of miR-185 in serum and placenta of patients with gestational diabetes mellitus. Clin Lab. 2019;65(12):12. doi:10.7754/Clin.Lab.2019.190445

72. Zhou X, Xiang C, Zheng X. miR-132 serves as a diagnostic biomarker in gestational diabetes mellitus and its regulatory effect on trophoblast cell viability. Diagn Pathol. 2019;14(1):119. doi:10.1186/ s13000-019-0899-9

73. Zhang L, Zhang T, Sun D, et al. Diagnostic value of dysregulated microribonucleic acids in the placenta and circulating exosomes in gestational diabetes mellitus. $J$ Diabetes Investig. 2021;12 (8):1490-1500. doi:10.1111/jdi.13493

74. Yu X, Liu Z, Fang J, Qi H. miR-96-5p: a potential diagnostic marker for gestational diabetes mellitus. Medicine. 2021;100(21):e25808. doi:10.1097/MD.0000000000025808

75. Msheik H, Azar J, El Sabeh M, Abou-Kheir W, Daoud G. HTR-8/ SVneo: a model for epithelial to mesenchymal transition in the human placenta. Placenta. 2020;90:90-97. doi:10.1016/j. placenta.2019.12.013

76. Uren RT, O’Hely M, Iyer S, et al. Disordered clusters of Bak dimers rupture mitochondria during apoptosis. Elife. 2017;6:e19944. doi:10.7554/eLife.19944

77. Sun DG, Tian S, Zhang L, et al. The miRNA-29b is downregulated in placenta during gestational diabetes mellitus and may alter placenta development by regulating trophoblast migration and invasion through a HIF3A-dependent mechanism. Front Endocrinol (Lausanne). 2020;11:169. doi:10.3389/fendo.2020.00169
78. Zhang C, Zhao D. MicroRNA-362-5p promotes the proliferation and inhibits apoptosis of trophoblast cells via targeting glutathione-disulfide reductase. Bioengineered. 2021;12 (1):2410-2419. doi:10.1080/21655979.2021.1933678

79. Stanirowski PJ, Szukiewicz D, Pazura-Turowska M, Sawicki W, Cendrowski K. Placental expression of glucose transporter proteins in pregnancies complicated by gestational and pregestational diabetes mellitus. Can J Diabetes. 2018;42(2):209-217. doi:10.1016/j. jcjd.2017.04.008

80. Hauguel-de Mouzon S, Shafrir E. Carbohydrate and fat metabolism and related hormonal regulation in normal and diabetic placenta. Placenta. 2001;22(7):619-627. doi:10.1053/plac.2001.0698

81. Khambule L, George JA. The role of inflammation in the development of GDM and the use of markers of inflammation in GDM screening. Adv Exp Med Biol. 2019;1134:217-242.

82. Yu J, Zhou Y, Gui J, Li AZ, Su XL, Feng L. Assessment of the number and function of macrophages in the placenta of gestational diabetes mellitus patients. J Huazhong Univ Sci Technolog Med Sci. 2013;33(5):725-729. doi:10.1007/s11596-013-1187-7

83. Sisino G, Bouckenooghe T, Aurientis S, Fontaine P, Storme L, Vambergue A. Diabetes during pregnancy influences Hofbauer cells, a subtype of placental macrophages, to acquire a pro-inflammatory phenotype. Biochim Biophys Acta. 2013;1832 (12):1959-1968. doi:10.1016/j.bbadis.2013.07.009

84. Nguyen-Ngo C, Jayabalan N, Salomon C, Lappas M. Molecular pathways disrupted by gestational diabetes mellitus. $J \mathrm{Mol}$ Endocrinol. 2019;63(3):R51-R72. doi:10.1530/JME-18-0274

85. Cao JL, Zhang L, Li J, et al. Up-regulation of miR-98 and unraveling regulatory mechanisms in gestational diabetes mellitus. Sci Rep. 2016;6(1):32268. doi:10.1038/srep32268

86. Zhao H, Tao S. MiRNA-221 protects islet beta cell function in gestational diabetes mellitus by targeting PAK1. Biochem Biophys Res Commun. 2019;520(1):218-224. doi:10.1016/j.bbrc.2019.09.139

\section{Publish your work in this journal}

Diabetes, Metabolic Syndrome and Obesity: Targets and Therapy is an international, peer-reviewed open-access journal committed to the rapid publication of the latest laboratory and clinical findings in the fields of diabetes, metabolic syndrome and obesity research. Original research, review, case reports, hypothesis formation, expert opinion and commentaries are all considered for publication. The manuscript management system is completely online and includes a very quick and fair peer-review system, which is all easy to use. Visit http://www.dovepress.com/testimonials.php to read real quotes from published authors. 